\title{
Registro de lesão por pressão: O que é abordado?
}

\author{
Dayse C. Nascimento, ${ }^{1}$ Gabriela A. S. Ferreira, ${ }^{2}$ Jéssica M. L. Silva, ${ }^{2 *}$ Marcelle T. Pioli, ${ }^{2}$ Monique R. M. Decio ${ }^{2}$
}

\section{Resumo}

Introdução: A lesão por pressão tem sido alvo de grande preocupação para os serviços de saúde, pois sua ocorrência causa impacto tanto para o paciente como para a sua família e a instituição, com aumento do tempo de internação. Objetivos: Levantar o que é abordado sobre registro de lesão por pressão nas bases científicas e analisar o que é desenvolvido nestes registros. Métodos: Revisão integrativa com coleta de dados através de um levantamento bibliográfico no Portal Regional da BVS, com os descritores "registros de enfermagem" e "úlcera por pressão", de documentos de 2011 a 2016, em português, inglês e espanhol e disponíveis na íntegra. Resultado: Foram obtidos 9 documentos, predominando o ano de 2014, originados dos EUA e em formato de artigo. Houve apenas uma abordagem superficial dos registros com utilização quantitativa para, por exemplo, dados epidemiológicos, notificações e indicador assistencial; pouco se aprofundou sobre o desenvolvimento de registros. Entretanto, houve relevância quanto ao registro à admissão do paciente, dada a importância financeira do registro, visto ser fonte de melhoria de comunicação e passível de tecnologias e da educação permanente. Conclusões: Há pouco registro qualitativo e descritivo das lesões por pressão, e há discrepância entre a importância do registro e sua prática. Sugere-se o uso de tecnologia e educação em saúde como ferramentas facilitadoras da prática do registro, uniformização de linguagem e comunicação, além de mais pesquisas descritivas sobre registro de lesão por pressão (LPP).

Descritores: Úlcera por pressão; Registros de enfermagem; Registro como assunto.

\section{Abstract}

\section{Pressure injury registry: what is covered?}

Introduction: Pressure injury has been a major concern for the health services, since its occurrence has an impact on both the patient and his/her family and for the institution, with an increase in the hospitalization time. Objectives: To raise what is written about pressure injury registry in the scientific bases, and to analyze what is developed in these registries. Method: Integrative review with data collection through a bibliographical survey in the Regional Portal of the VHL, with the descriptors "Nursing records" and "pressure ulcer", documents from 2011 to 2016, in Portuguese, English and Spanish, and available in full. Results: There was 9 documents, predominating the year 2014, originated in the USA and in an article format. Conclusions: There was only a superficial approach of the records with quantitative use in the articles for, for example, epidemiological data, notifications and care indicator, little progress has been made on the development of
1. Comissão de Curativos. Hospital Universitário Pedro Ernesto. Universidade do Estado do Rio de Janeiro. Rio de Janeiro, RJ, Brasil.

2. Especialização em Estomaterapia. Universidade do Estado do Rio de Janeiro. Rio de Janeiro, RJ, Brasil.

*Endereço para correspondência:

Boulevard Vinte e oito de setembro, 77, prédio anexo, sl. 2

Rio de Janeiro, RJ, Brasil. CEP: 20551-030.

E-mail: jessica-mesquitals@hotmail.com

Revista HUPE, Rio de Janeiro, 2016;15(4):343-348

doi: 10.12957/rhupe.2016.31614

Recebido em 22/05/2017. Aprovado em 23/07/2017.

records. However, there was relevance regarding registration of patient admission, due to the financial importance of the registry, as it is source of communication improvement and subject to technology and permanent education. Conclusions: There is little qualitative and descriptive record of pressure injuries, and there is a discrepancy between the importance of the registry and its practice. We suggest the use of technology and health education as tools to facilitate the practice of registration, standardization of language and communication, and a more descriptive research on LPP registration.

Keywords: Pressure ulcer; Nursing record; Records as topic.

\section{Resumen}

\section{Registro de la lesión por presión: Qué está cubierto?}

Introducción: La lesión por presión ha sido de gran interés para orientar los servicios de salud debido a sus impactos de la ocurrencia tanto para el paciente y su familia y la institución, con una mayor duración de la estancia. Objetivos: Recaudar lo que está cubierto bajo el registro de lesiones presión en bases científicas, y analizar lo que se desarrolla en estos registros. Método: Revisión integrada con la recogida de datos a través de una revisión de la literatura en el Portal Regional de la BVS, con los descriptores "registros de enfermería" y "úlceras por presión" desde 2011 hasta 2016 documentos en portugués, Inglés y Español, y disponible en su totalidad. Resultado:Se obtuvieron 9 documentos, predominantemente el año 2014, los EE.UU. su origen y formato de artículo. Resultados: Sólo hubo un acercamiento superficial a los registros con el uso cuantitativo de los artículos, por ejemplo, los datos epidemiológicos, las notificaciones y poco indicador de bienestar profundizado en el desarrollo de registros. Sin embargo, hubo relevancia como para grabar en la admisión, la importancia financiera registro, que és fuente de mejora de la comunicación y capaz 


\section{Artigo de revisão}

de tecnologías y educación continua. Conclusiónes: Hay poca registro cualitativo y descriptivo de lesiones por presión, y no hay discrepancia entre la importancia de la grabación y la práctica. Se sugiere el uso de la tecnología y la educación sanitaria como herramientas que facilitan la práctica de re-

\section{Introdução}

Uma das consequências mais comuns, resultante de longa permanência em hospitais, é o aparecimento de alterações da pele. Isso é evidenciado diante da combinação de fatores de risco, como idade avançada, comorbidades e imobilidade. A manutenção da integridade da pele dos pacientes internados tem por base o conhecimento de que medidas de cuidado relativamente simples podem garantir que a pele do paciente se mantenha íntegra durante seu período de internação. A maioria das recomendações para avaliação e medidas preventivas pode ser utilizada de maneira universal, ou seja, tem validade tanto para prevenção de lesão por pressão (LPP) como para quaisquer outras alterações da pele.

Diferentemente de boa parte das alterações da pele, a LPP tem sido alvo de grande preocupação para os serviços de saúde, pois sua ocorrência causa impacto tanto para os pacientes como para suas famílias e instituição, com aumento do tempo de internação. Nessa concepção, a LPP se apresenta como importante desafio, visto ser reconhecida como um evento adverso quando ocorre após a admissão do indivíduo no serviço de saúde, além de ser vista como uma das cinco causas mais comuns de danos aos pacientes.

LPPs ainda são consideradas como problema grave e causam complicações de ordem emocional aos pacientes hospitalizados ou à comunidade. A pessoa com tal ferida pode apresentar também sofrimento e dor, além de afastamento social.E ainda, representa um desafio permanente para os profissionais de saúde, pacientes e instituições devido a sua elevada incidência e prevalência, assim como os altos índices de mortalidade e morbidade, além de custos gerados. ${ }^{2}$

Em 2016, foi redefinida a nomenclatura de úlcera por pressão para lesão por pressão (LPP) e a atualização da nomenclatura dos estágios do sistema de classificação. O termo expressa de forma mais clara esse tipo de lesão, tanto na pele sem danos quanto na pele ulcerada. No antigo sistema do consenso citado, o Estágio 1 e a Lesão Tissular Profunda retratavam lesões em pele preservada à medida que as categorias descreviam lesões abertas. Com isso, gerava um equívoco porque a descrição de cada estágio reporta-se à úlcera por pressão. ${ }^{3}$ gistro, la normalización de la lengua y la comunicación, y la investigación más descriptivo en el registro LPP.

Palabras clave: Úlcera por pressíon; Registro de enfermería; Registros como assunto.

Além dessa alteração, no novo acordo, os algarismos romanos não serão utilizados na nomenclatura de estágio, passando a ser empregados, os arábicos. A palavra suspeita foi retirada da categoria diagnóstica Lesão Tissular Profunda. Também foram adicionadas outras definiç̧ões de lesões por pressão: Lesão por Pressão Relacionada a Dispositivo Médico e Lesão por Pressão em Membrana Mucosa. ${ }^{3}$

As escalas de avaliação de risco estabelecem, por meio de pontuação, a probabilidade da ocorrência da LPP em um paciente, com base numa série de parâmetros considerados como fatores de risco. A utilização das escalas de classificação de riscos (escalas preditivas), mudança de decúbito e suporte nutricional são algumas estratégias assistenciais para prevenção de LPP.

Assim, é possível avaliar e reconhecer pacientes que poderão desenvolvê-la. Por meio de uma pontuação, é possível avaliar a possibilidade de ocorrência da LPP, com base em parâmetros considerados como fatores de risco. Estas escalas incluem condição geral e avaliação da pele, mobilidade, umidade, incontinência, nutrição, dor, entre outros fatores, sendo as mais utilizadas a de Norton, de Waterlow e de Braden, diferindo na abrangência, complexidade e aplicabilidade. ${ }^{4,5}$

As escalas têm muita utilidade, pois se complementam e favorecem a avaliação sistemática do paciente. Nos pacientes complexos, estes instrumentos devem ser aplicados diariamente, devido à instabilidade clínica. Por isso eles são indispensáveis à adoção de procedimentos de prevenção após o diagnóstico do risco. O desempenho do enfermeiro na avaliação do risco é muito importante porque promove um cuidado completo e individualizado ao paciente e à família, proporcionando informações imprescindíveis para o plano de cuidado e garantindo comunicação multidisciplinar eficiente.

As lesões por pressão acometem pacientes em diversos cenários do cuidado da enfermagem. Para entender melhor a problemática é necessário conhecer sua incidência e prevalência. A prevalência de lesão de pressão em instituições dos Estados Unidos oscila de 3\% a 14\%, subindo para 15\% a 25\% nas situações em que os enfermos estão acamados. ${ }^{6,7}$ Em um dos poucos estudos 
nacionais sobre o tema, envolvendo pacientes hospitalizados em serviço universitário do interior paulista, o índice de 0,94\% apresentado é considerado bastante baixo comparativamente aos índices internacionais.

A incidência e prevalência das lesões por pressão são usadas como um dos precursores para estabelecer a qualidade da assistência oferecida nas instituições de saúde, visto que cerca de $95 \%$ das lesões por pressão são controladas com a aplicação de ações especiais. Por isso, a identificação imediata de pacientes em risco de evolução por meio de um método validado possibilita a adesão de critérios preventivos. Entretanto, não adianta adotar e aplicar uma estratégia de avaliação de riscos produtiva de alta sensibilidade e especificidade para o acompanhamento da LPP se os dados relacionados a esse evento adverso (EA) não forem corretamente anotados. ${ }^{8}$

O prontuário do paciente reúne informações como exames e condutas realizados pelos profissionais de saúde. A falta de registro ou o registro incorreto em prontuário prejudica o seguimento do cuidado prestado em suas diversas etapas, o planejamento assistencial da equipe multiprofissional e a segurança do paciente. A comunicação pode acontecer no hospital de diferentes formas, seja pela linguagem verbal ou escrita, as quais auxiliam o acolhimento dos pacientes. Desse modo, o diálogo deve ser contínuo entre os profissionais de saúde para que as necessidades dos pacientes sejam acolhidas. Para uma boa comunicação, é necessário registro no prontuário, pois é a principal forma de comunicação entre os profissionais de saúde sobre o paciente. ${ }^{9}$

Cabe ressaltar também que, falando nos termos da lei, é responsabilidade e dever do profissional de enfermagem registrar em meios apropriados as informações emergentes do cuidado e do gerenciamento dos processos de trabalho. Assim, para que seja possível assegurar a continuidade e qualidade da assistência, é fundamental a composição desse registro por coleta de dados, diagnóstico de enfermagem, intervenções de enfermagem e resultados alcançados, além da identificação do profissional com sua assinatura. ${ }^{10} \mathrm{O}$ registro das informações inerentes e indispensáveis ao processo de cuidar de forma clara, objetiva e completa é uma responsabilidade do profissional, não somente com o paciente, mas também com a família, a comunidade e com a organização empregadora. ${ }^{10,11}$

Além da importância do registro, especificamente das feridas, para analisarmos sua evolução, há necessidade de itens e parâmetros para avaliar não só a conduta desenvolvida, como a resposta a essa conduta aplicada.
Itens importantes para a classificação das feridas como a etiologia, tempo de instalação da lesão, complexidade, comprometimento tecidual, além de parâmetros fundamentais para a monitorização do estado da lesão: tamanho da lesão, aspecto da pele perilesional, exsudato (presença, característica, quantidade), dor, infecção (presença, patógeno), e tipo de tecido encontrado no leito são fundamentais. ${ }^{6,12}$ Ademais, tais itens servem de avaliação em diversos instrumentos como: "Avaliação de Feridas", 13 "Escala de Push". ${ }^{14}$

Considerando, então, a importância do registro para o desenvolvimento da prática científica do tratamento de lesões por pressão e sua necessidade para acompanhamento e tratamento dessas feridas, faz-se importante a análise nas bases científicas da abordagem ao registro e uma possível uniformização e direcionamento de registro realizado na prática. Assim, como objeto deste estudo está o registro de enfermagem sobre lesão por pressão, e como objetivos planejamos levantar o que é abordado sobre registro de lesão por pressão nas bases científicas e analisar o que é desenvolvido nestes registros.

\section{Metodologia}

O estudo é uma revisão integrativa por meio de coleta de dados realizada a partir da produção acadêmica do campo da enfermagem, através de um levantamento bibliográfico ancorado nos objetivos das autoras para responder as questões formuladas por meio de sua vivência profissional. A pesquisa bibliográfica é feita a partir do levantamento de referências teóricas já analisadas, e publicadas por meios escritos e eletrônicos, como artigos científicos, dissertações e teses. Este tipo de pesquisa é parte importante do processo de recolhimento de informações ou conhecimentos prévios sobre o problema a respeito do qual se procura a resposta. ${ }^{15}$

Para realização desse trabalho de natureza qualitativa sobre os registros de enfermagem sobre LPP, foi realizado o levantamento no Portal Regional da BVS, na área de busca avançada, a partir dos seguintes descritores: "registros de Enfermagem" e "úlcera por pressão", utilizados simultaneamente e acompanhados do operador booleano "and". Os descritores foram conferidos no Portal de Descritores em Ciências da Saúde (DeCS). O resultado inicial foi de 206 documentos.

Após o levantamento, foram aplicados os filtros: texto completo disponível; seleção dos anos de 2011 a 2016, sendo esses os cinco anos mais recentes próximos a elaboração deste trabalho; idiomas de português, inglês e espanhol; chegando ao pré-resultado de 25 docu- 


\section{Artigo de revisão}

mentos. Para análise, foram considerados todos os tipos de documentos achados. Os levantamentos decorreram de 01 de novembro de 2016 até 05 de dezembro de 2016.

Os critérios de inclusão utilizados para a busca foram: artigos publicados em português, inglês e espanhol, artigos na íntegra que retratassem a temática referente à revisão integrativa e artigos publicados e indexados nos referidos bancos de dados. E os critérios de exclusão foram: artigos que não pertencem ao campo de estudo da enfermagem, artigos que não tivessem acesso na íntegra, artigos indexados com duplicidade em diferente banco de dados.

A fim de organizar o material pesquisado, elaborou-se um instrumento de coleta de dados, (Apêndice A) baseado nas seguintes categorias: ano de publicação, título da publicação, autores, tipo de publicação, origem (país), abordagem ao objeto.

A análise dos dados coletados foi realizada na perspectiva de análise de conteúdo. Essa técnica permite a reprodução e validação de inferências sobre dados de um determinado contexto, utilizando procedimentos especializados e específicos. Este tipo de tratamento de dados assemelha-se à lógica dos estudos quantitativos, uma vez que permite a interpretação cifrada do material qualitativo. ${ }^{16,17}$ Esta técnica se divide em três fases: 1) pré-análise, 2) exploração do material e 3) tratamento dos resultados, inferência e interpretação. ${ }^{16}$

Dos 25 documentos apontados nesse levantamento, considerando que ainda que tenha sido utilizado o filtro de "disponibilidade na íntegra", a tentativa de leitura superficial, alguns documentos exigiam cadastros/associações/assinaturas para acesso, por isso foram retirados da pesquisa.

Além destes, também foram removidas as repetições de documentos, assim como os documentos que não continham a unidade de análise: registro, ou itens semelhantes que correspondessem ao registro ou documentação em meio específico das lesões por pressão. Assim, o total do resultado para análise foi de 9 documentos, e esses foram tabulados quanto à frequência de ano de publicação, os tipos dos documentos levantados e o país de origem, que apresentaram dados mais densos (Tabelas 1 a 3). E posteriormente, deu-se a abordagem qualitativa já citada anteriormente.

\section{Resultados}

Como destaque abordável houve predominância de documentos do ano de 2014, originados dos EUA e em formato de artigo. Tal evidência pode estar associada ao fato de que um dos maiores órgãos de abordagem à lesão por pressão, National Pressure Ulcer Advisory Panel (NPUAP), reconhecido internacionalmente, foi criado na capital dos Estados Unidos, podendo então haver uma influência local de produção científica acerca do tema; e, em 2014, houve uma atualização sobre, ainda no termo antigo, úlcera por pressão, com documentos traduzidos para diversos idiomas com acesso a vários localidades, podendo também ser uma influência para a produção em tal ano. ${ }^{3}$

\section{Discussão}

Um dos dados importantes achados neste levantamento foi a necessidade do registro da existência da lesão por pressão(LPP) e do risco de desenvolvimento de lesão por pressão na admissão do paciente na unidade assistencial. Há instituições que norteiam o reembolso financeiro dos custos com o tratamento da LPP em função do tempo de desenvolvimento desta. Ou seja, não se reembolsa quando essa ferida se instalou durante a internação do paciente. Assim, se demonstra a importância da notificação inicial para direcionamento de condutas e custeios.

Tabela 1. Distribuição dos artigos por ano de publicação

\begin{tabular}{c|c|c}
\hline ANO & Fi & Fi(\%) \\
\hline 2016 & 2 & $22,22 \%$ \\
2014 & 3 & $33,34 \%$ \\
2013 & 2 & $22,22 \%$ \\
2012 & 2 & $22,22 \%$ \\
\hline
\end{tabular}

Tabela 2. Distribuição dos artigos por tipo de publicação

\begin{tabular}{c|c|c}
\hline TIPO DE DOCUMENTO & Fi & Fi(\%) \\
\hline Artigo & 7 & $77,78 \%$ \\
\hline Monografia & 1 & $11,11 \%$ \\
\hline Artigo editorial & 1 & $11,11 \%$ \\
\hline Total & 9 & $100 \%$ \\
\hline
\end{tabular}

Tabela 3. Distribuição dos artigos por localidade

\begin{tabular}{c|c|c}
\hline Local & $\mathbf{F i}$ & $\mathbf{F i ( \% )}$ \\
\hline EUA & 5 & $44,45 \%$ \\
Brasil & 3 & $33,33 \%$ \\
\hline Peru & 1 & $11,11 \%$ \\
Espanha & 1 & $11,11 \%$ \\
Total & 9 & $100 \%$ \\
\hline
\end{tabular}


Além deste, com fundamental registro à internação do paciente, há também a classificação do risco de desenvolvimento de LPP para direcionamento precoce de condutas preventivas. Deve-se existir a valorização e identificação de fatores de risco registrando-os com um melhor norteador da assistência. . $1,19^{19}$

Outro achado abordava a questão dos registros nas evoluções de enfermagem como fonte de coleta de dados para análise, e principalmente como indicador da qualidade da assistência. ${ }^{20,21}$ Cabe ressaltar a importância da prevenção da LPP como um dos itens de programas de segurança do paciente, porém, deve-se notificar e registrar para possível análise da assistência e direcionamento de práticas preventivas. Porém, este só pode ser feito se houver registro em documentos próprios dos pacientes, e também, a notificação de tais eventos. Além disso, as pesquisas para alimentação epidemiológica sobre LPP só podem ser feitas a partir da quantificação dos dados presentes, então, precisa-se notificar e registrar. ${ }^{20}$

Assim, a ausência de registro não só enfraquece as pesquisas sobre o tema, como desqualifica principalmente a assistência. Em um dos artigos se notava inclusive a dissociação entre a história e o estado clínico do paciente, a realidade documentada pelos profissionais do setor e a realidade observada por uma profissional especialista (estomaterapeuta). Neste artigo se identificou escassez de dados fidedignos registrados, além de um número maior de lesões evidenciadas pela observação de uma especialista. ${ }^{22}$

Houve ainda registros pouco mais descritivos, com relato de localização das feridas, tamanho, quantidade, tipo de tecido, porém a frequência desses dados era menor. Esta realidade foi comprovada com mais dois estudos, um dos quais foi registrada somente a localização das lesões estava pontuada, e no outro, o tamanho e o estadiamento, porém sem desdobramento ou abordagem minuciosa dos registros identificados nos artigos. ${ }^{23,24}$

Pode-se destacar também que em um dos estudos, em que havia um fluxograma institucional para direcionamento de condutas, havia a referência para a especialista estomaterapeuta. Este estudo também demonstrou melhor observação e avaliação das lesões, quando comparado a profissionais de formação geral. 22,24

Entretanto, considerando a existência de profissionais de diversas especialidades na realidade prática, além das grandes demandas operacionais e gerenciais, o registro se apresenta como instrumento facilitador, com documentos uniformes e próprios para notificação e registro contínuo de LPP..$^{18}$ Nesse contexto a tecnologia é evidenciada como um aliado ao registro, 20,25,26 pois facilitaria a indexação de informações, e também poderia facilitar na comunicação entre profissionais, além da redução do tempo para registro.

Tão fundamental quanto os itens citados, a educação contínua e capacitação profissional sobre o reconhecimento das lesões e termos associados ajudariam a prática do registro. Não apenas uniformizando a linguagem e melhorando a comunicação entre profissionais, como também proporcionando o encorajamento ao profissional para registrar sua observação clínica e prática profissional captadas no dia a dia. ${ }^{18,20}$

\section{Conclusão}

Conclui-se neste estudo que há uma carência considerável no desenvolvimento qualitativo descritivo acerca das LPP, com dois estudos pontuando algumas características destas feridas. Entretanto, esses estudos não abordam sua evolução. Juntamente com as funções já citadas inicialmente neste trabalho, o registro tem importância para além da assistência diretamente aplicada como indicador assistencial, ou seja, ele também é fundamental para as necessidades quantitativas da epidemiologia da LPP, além da notificação, manejo financeiro da assistência, classificação de risco à admissão. Por fim, é considerado objeto de comunicação e uniformização da linguagem assistencial, de questões tecnológicas e da educação permanente.

Assim, como sugestão, para adesão dos profissionais à pratica do registro, destacam-se estratégias que facilitem o registro, como o uso da tecnologia que aproxime o profissional da prática de registrar. Além da ênfase para a importância do registro como benefício à comunicação entre profissionais, indicador de qualidade assistencial e norteador de condutas, partindo do princípio da continuidade da prática de registrar. $\mathrm{E}$ também, a capacitação profissional como uma ferramenta fundamental para o alcance dessas ratificações.

Como limitações deste estudo, se destacou a grande quantidade de artigos restritos as assinaturas para acesso desde o levantamento inicial até a pré-análise, o que gera perda de documentos no desenvolvimento do trabalho. Além disso, houve pouco registro qualitativo das lesões por pressão, sugerindo mais estudos sobre o 


\section{Artigo de revisão}

desenvolvimento qualitativo e descritivo das lesões por pressão da prática diária.

\section{Referências}

1. Vasconcelos JMB, Caliri MHL. Ações de enfermagem antes e após um protocolo de prevenção de lesões por pressão em terapia intensiva. Esc. Anna Nery 2017;21(1):e20170001.

2. Ferreira JDL, Aguiar ESS, Soares MJGO, et al. Risco e prevalência de úlcera por pressão em usuários da rede básica de saúde. J Nurs UFPE on line. 2012 Sept;6(9):2045-51

3. European Pressure Ulcer Advisory Panel and National Pressure Ulcer Advisory Panel. Prevention and treatment of pressure ulcers: quick reference guide. Washington DC: National Pressure Ulcer Advisory Panel;2014.

4. Borghardt AT, Araújo TM, Rogenski NMB, et al. Avaliação das escalas de risco para úlcera por pressão em pacientes críticos: uma coorte prospectiva. Rev. Latino-Am. Enfermagem. jan.fev. 2015;23(1):28-35

5. Santos MP, Neves RC, Santos CO. Escalas utilizadas para prevenir úlceras por pressão em Pacientes críticos. Revista Enfermagem Contemporânea. 2013;2(1):19-31.

6. Blanes L, Duarte IS, Calil JA, et al. Avaliação Clínica e epidemiológica das úlceras por pressão em pacientes internados no Hospital São Paulo. Rev Assoc Med Bras. 2004;50(2):182-7

7. Rogenski NMB, Santos VLCG. Estudo sobre a incidência de úlceras por pressão em um hospital universitário. Rev Latino-Am Enfermagem. 2005 julho-agosto;3(4):474-80

8. Brasil. Ministério da Saúde. Documento de referência para o Programa Nacional de Segurança do Paciente / Ministério da Saúde; Fundação Oswaldo Cruz; Agência Nacional de Vigilância Sanitária. - Brasília: Ministério da Saúde, 2014. 40 p.

9. Pedrosa KKA, Souza MFG, Monteiro A. O enfermeiro e 0 registro de enfermagem em um hospital público de ensino. Rev Rene, Fortaleza. 2011 jul/set;12(3):568-73.

10. COFEN. Resolução n. 429, de 30 de maio de 2012. Registro das ações profissionais no prontuário do paciente, e em outros documentos próprios da enfermagem, independente do meio de suporte - tradicional ou eletrônico. Disponível em www. cofen.gov.br/resoluo-cofen-n-4292012_9263.html

11. COFEN. Resolução $n^{\circ}$. 311, de 08 de fevereiro de 2007. Aprova a Reformulação do Código de Ética dos Profissionais de Enfermagem. Disponível em: www.cofen.gov.br/resoluo-cofen-3112007_4345.html

12. Geovanini T. Tratado de feridas e curativos: Enfoque multi- profissional. São Paulo: Editora Rideel; 2014

13. Bajay HM, Araújo IEM. Validação e confiabilidade de um instrumento de avaliação de feridas. Acta Paul. Enferm. 2006 July/ Sept;19(3):290-95.

14. Santos VLCG. Adaptação transcultural do pressure ulcer scale for healing (push) para a língua portuguesa. Rev Latino-am Enfermagem 2005 maio-junho;13(3):305-13.

15. Fonseca JJS. Metodologia da pesquisa científica. Fortaleza: UEC, 2002. Apostila.

16. Bardin L. Análise de conteúdo. São Paulo: Edições 70, 2011. p.15-59.

17. Minayo MCS. O desafio do conhecimento: pesquisa qualitativa em saúde. São Paulo: Ed. Hucitec, 2013. p.303-319.

18. Rogers C. Improving processes to capture present-on-admission pressure ulcers. Adv Skin Wound Care. 2013 Dec;26(12):566-72.

19. Bustamante MLC. Factores que limitan la práctica de cuidados preventivos de úlceras por presión en la Unidad de Cuidados Críticos del Hospital Nacional Guillermo Almenara Irigoyen 2009. [Monografia] Peru. Universidad Nacional Mayor de San Marcos. 2014

20. Santos CT, Oliveira MC, Pereira AGS, et al. Indicador de qualidade assistencial úlcera por pressão: análise de prontuário e de notificação de incidente. Rev Gaúcha Enferm. 2013;34(1):111-118.

21. Quadros DV, Magalhães AMM, Mantovani VM, et al. Análise de indicadores gerenciais e assistenciais após adequação de pessoal de enfermagem. Rev Bras Enferm [Internet]. 2016;69(4):638-43.

22. Sebastián-Viana T, González-Ruiz JM, Núñez-Crespo J, et al. La validez de un registro clínico de úlceras por presión. Anales Sis San Navarra Pamplona. 2014 ene./abr; (37):1.

23. Sousa LRM, Bezerra SMG, Mesquita RF, et al. Análise da prevalência de desbridamento cirúrgico de úlcera por pressão em um hospital municipal. J. res.: fundam. care. online 2016. abr./ jun. 8(2):4186-4196.

24. Estilo ME, Angeles A, Perez T, et al. Pressure ulcers in the intensive care unit: new perspectives on an old problem. Crit Care Nurse. 2012 Jun; 32(3):65-70.

25. Kim H, Chung H, Wang S, et al. SAPPIRE: a prototype mobile tool for pressure ulcer risk assessment. Stud Health Technol Inform. 2014;201:433-40.

26. Dowding DW, Turley M, Garrido T. The impact of an electronic health record on nurse sensitive patient outcomes: an interrupted time series analysis. J Am Med Inform Assoc. 2012 Jul-Aug;19(4):615-20. 